Pieper, M.J.C., Francke, A.L., Steen, J.T. van der, Scherder, E.J.A., Twisk, J.W.R., Kovach, C.R Achterberg, W.P. Effects of a stepwise multidisciplinary intervention for challenging behavior in ) advanced dementia: a cluster randomized controlled trial. Journal of the American Geriatries 7 Society: 2016, 64(2), 261-269

\begin{tabular}{|l|l|}
\hline $\begin{array}{l}\text { Postprint } \\
\text { Version }\end{array}$ & 1.0 \\
\hline Journal website & $\frac{\text { http://onlinelibrary.wiley.com/doi/10.1111/igs.13868/abstract;jsessionid=A16294E }}{\underline{\text { OBD91C3A20D8DB475D87A1F59.f01t04 }}}$ \\
\hline Pubmed link & $\underline{\text { http://www.ncbi.nlm.nih.gov/pubmed/26804064 }}$ \\
\hline DOI & $10.1111 /$ jgs.13868 \\
\hline
\end{tabular}

This is a NIVEL certified Post Print, more info at http://www.nivel.eu

\title{
Effects of a Stepwise Multidisciplinary Intervention for Challenging Behavior in Advanced Dementia: A Cluster Randomized Controlled Trial
}

MARJOLEINE J. C. PIEPER MSC ${ }^{1-2-3}$, ANNEKE L. FRANCKE PHD ${ }^{1-4}$, JENNY T. VAN DER STEEN PHD ${ }^{1-2}$, ERIK J. A. SCHERDER PHD ${ }^{5}$, JOS W. R. TWISK PHD ${ }^{6}$, CHRISTINE R. KOVACH PHD ${ }^{7}$, WILCO P. ACHTERBERG PHD ${ }^{1-3}$

${ }^{1}$ Emgo Institute for Health and Care Research, Amsterdam, The Netherlands

2 Department of General Practice and Elderly Care Medicine, VU University Medical Center Amsterdam, Amsterdam, The Netherlands

${ }^{3}$ Department of Public Health and Primary Care, Leiden University Medical Center, Leiden, The Netherlands

${ }^{4}$ Netherlands Institute for Health Services Research, Utrecht, The Netherlands

${ }^{5}$ Department of Clinical Neuropsychology, VU University Amsterdam, Amsterdam, The Netherlands

${ }^{6}$ Department of Epidemiology and Biostatistics, VU University Medical Center Amsterdam, Amsterdam, the Netherlands

${ }^{7}$ University of Wisconsin-Milwaukee, Milwaukee, Wisconsin

\begin{abstract}
Objectives: To assess whether implementation of a stepwise multicomponent intervention (STA OP!) is effective in reducing challenging behavior and depression in nursing home residents with advanced dementia.

Design: Cluster randomized controlled trial.

Setting: Twenty-one clusters (single independent nursing home units) in 12 nursing homes within the Netherlands.

Participants: Residents with advanced dementia $(\mathrm{N}=288)$.

Intervention: Staff working on intervention units received comprehensive stepwise multidisciplinary training; the control condition received training on general nursing skills, dementia management and pain without the stepwise component.

Measurements: The primary outcome was agitation (Cohen-Mansfield Agitation Inventory (CMAI)). Secondary outcomes included psychotropic medication use, neuropsychiatric symptoms (Neuropsychiatric Inventory-Nursing Home version (NPI-NH)), and symptoms of depression (Cornell Scale for Depression in Dementia (CSDD), Minimum Dataset Depression Rating Scale (MDS-DRS)).
\end{abstract}


Pieper, M.J.C., Francke, A.L., Steen, J.T. van der, Scherder, E.J.A., Twisk, J.W.R., Kovach, C.R Achterberg, W.P. Effects of a stepwise multidisciplinary intervention for challenging behavior in ) advanced dementia: a cluster randomized controlled trial. Journal of the American Geriatries 7 Society: 2016, 64(2), 261-269

Measurements were made at baseline and 3 and 6 months after the intervention. Multilevel analysis and logistical generalized estimating equations were used to test treatment and time effects. Analysis was on an intention-to-treat basis.

Results: Multilevel modeling revealed an overall effect of the intervention on challenging behavior and depression; CMAI (mean difference -4.07 points, $95 \%$ confidence interval $=(\mathrm{CI})=-7.90$ to $-0.24, P=.02)$, NPI-NH (mean difference -3.57 points, $95 \% \mathrm{CI}=-6.30$ to $-0.84, P=.005$ ), CSDD (mean difference -1.59 points, $95 \% \mathrm{CI}=-2.49$ to $-0.69, P<.001$ ), and MDS-DRS (mean difference -0.96 points, $95 \% \mathrm{CI}=-1.40$ to $-0.52, P<.001$ ) scores were significantly lower in the intervention condition than the control condition. There was a significant reduction of antidepressants $(\mathrm{N} 06 \mathrm{~A})(\mathrm{OR}=0.32)$; nonsignificant reductions of antipsychotics (N05A), anxiolytics (N05B), and hypnotic-sedatives (N05C) (odds ratios $=0.69$ to 0.90 ).

Conclusion: For nursing home residents with advanced dementia and challenging behavior, providing staff with comprehensive training in behavioral management, resulted in improved behavior and less psychotropic medication use.

Neuropsychiatric symptoms are highly prevalent in advanced dementia. Symptoms include psychotic features such as hallucinations and delusions, depression, aggressive and agitated behavior, euphoria, and apathy.[1] Together with physical impairment, neuropsychiatric symptoms have a major effect on quality of life in individuals with dementia and are the main reason for seeking help and institutionalization.[2-4] One study reported that $97 \%$ of nursing home residents with dementia had at least one neuropsychiatric symptom over a 2-year period.[5] These problems are especially prominent and burdensome in the more-advanced stages of dementia and are usually distressing for the individuals, their family, (in)formal caregivers, and treating physicians (e.g., neurologists, psychiatrists, geriatricians).[4] Neuropsychiatric symptoms also frequently lead to the prescription of psychotropic medications.[6] Although such medications have their place in the treatment of severe or persistent psychiatric symptoms, they are associated with substantial side effects, including falls, cerebrovascular events, and mortality. The etiology of neuropsychiatric symptoms in dementia is probably multifactorial and includes neuropathological changes in the brain related to dementia, and unmet physical (e.g., pain) and psychological needs.[7] Many interventions (psychosocial and pharmacological) that address these needs have been investigated; their goals vary widely depending on the behavioral expression of the unmet need. $[8,9]$ One of the few interventions that acknowledges this complex etiology and combines psychosocial and pharmacological interventions for challenging behavior is the Serial Trial Intervention (STI).[10] This intervention (applying a stepwise protocol) was successful in decreasing discomfort in individuals with dementia in nursing homes in the United States. Specific targets of that study were decreasing discomfort, and returning challenging behavior to normal. Despite these positive results, a limitation of that study is that the effects of the intervention on a broader range of neuropsychiatric symptoms, including depression, were not established. Moreover, possible cluster effects were not taken into account in the analysis. These limitations, 
Pieper, M.J.C., Francke, A.L., Steen, J.T. van der, Scherder, E.J.A., Twisk, J.W.R., Kovach, C.R Achterberg, W.P. Effects of a stepwise multidisciplinary intervention for challenging behavior in ) advanced dementia: a cluster randomized controlled trial. Journal of the American Geriatries 7 Society: 2016, 64(2), 261-269

plus the fact that the organization, availability, and level of education of staff (and the availability of additional resources) differ across settings and countries,[11, 12] led to uncertainty that these positive results would be maintained crossculturally.[13]

Therefore, to evaluate the STI in this setting and to include additional neuropsychiatric symptoms, the STI was translated and adjusted, and a cluster randomized controlled trial (STA OP!)[13] was conducted to assess whether implementation of this redesigned intervention would lead to fewer expressions of challenging behavior, better mood, and less use of antipsychotics (resulting in fewer side effects).

\section{MATERIAL AND Methods}

\section{Study Design and Participants}

The STA OP! trial is a cluster randomized controlled trial. The recruitment strategy, participant samples, study protocol,[13] and the outcomes of pain and quality of life are described elsewhere. In brief, participating nursing homes were recruited from within the academic nursing home network of the VU University Medical Center[14] and met the criteria, that at least one psychogeriatric unit was willing to participate and no major organizational changes or building activities were planned or performed during the study period. In each nursing home, residents with moderate to severe cognitive impairment (Reisberg Global Deterioration Scale (GDS) Stage 5, 6, or 7),[15] no psychiatric diagnosis other than dementia, and clinically significant symptoms of challenging behavior (Neuropsychiatric Inventory-Nursing Home version (NPI-NH) score $>4$ or Cohen-Mansfield Agitation Inventory (CMAI) score $>44)[16,17]$ were eligible for participation, providing that written proxy consent was received.

\section{Randomization, Masking, and Ethical Approval}

The trial was single blinded (the researcher knew the condition, but the research assistants performing the measurements were blinded).[13] Residents were the targets of the intervention, but because the intervention was multidisciplinary, and training was given to the entire staff of a nursing home unit, the nursing home units served as the unit of randomization to minimize contamination and carryover effects. An independent researcher (who was unaware of the identity of the units) performed the allocation using a computer-generated sequence program (Random Allocation Software, EMGO+ Institute, Amsterdam, the Netherlands). Data collection was performed between January 2010 and June 2012. Approval was obtained from the medical ethics review committee of the VU University Medical Center (VUmc number 2009/119). This trial is registered at the Netherlands National Trial Register (NTR1967).

\section{Procedures}

A trained research assistant assessed all measures based on a face-to-face interview with the nursing staff member (a certified nursing assistant or registered nurse) who was familiar with the resident before implementation and 3 (end of the training period) and 6 months after the intervention. Symptoms of challenging behavior were recorded using the CMAI and a Dutch translation of the NPI-NH. The primary outcome measure was the CMAI, a 29-item instrument (score range 29-203) with good validity and reliability $[18,19]$ used to assess agitation in nursing home 
Pieper, M.J.C., Francke, A.L., Steen, J.T. van der, Scherder, E.J.A., Twisk, J.W.R., Kovach, C.R Achterberg, W.P. Effects of a stepwise multidisciplinary intervention for challenging behavior in ) advanced dementia: a cluster randomized controlled trial. Journal of the American Geriatries 7 Society: 2016, 64(2), 261-269

residents with dementia.[16, 18, 20] The NPI-NH, a structured interview that nivel includes 12 items to rate the frequency and severity of neuropsychiatric symptoms in residents with dementia[17,21] (delusions, hallucinations, agitation or aggression, depression or dysphoria, anxiety, euphoria, apathy, disinhibition, irritability or lability, aberrant motor behavior, nighttime behavior disturbances, appetite or eating abnormalities) was used for measurement of neuropsychiatric symptoms. The score for each item ranges from 0 to 12 , with higher scores indicating worse behavior. The multiplication of frequency times severity scores results in a total score ranging from 0 to 144 . The Dutch version of the NPI-NH is reported to have good validity and reliability.[21, 22]

Symptoms of depression were recorded using the Cornell Scale for Depression in Dementia (CSDD),[23] a 19-item instrument, with scores for each item ranging from 0 to 2 (total score range 0-38), and the Minimum Dataset Depression Rating Scale (MDS-DRS),[24] a seven-item instrument rating the presence of depressive symptoms in the past 30 days. Scores range from 0 to 14, with higher scores indicating more depressive symptoms. Both depression scales have been validated for use in individuals with depression and dementia.[23, 24]

Psychotropic drug use was recorded and retrieved from medication lists and classified with the Anatomical Therapeutic Chemical (ATC) classification.[25] The drugs were grouped into antipsychotics (N05A), anxiolytics (N05B), hypnotics and sedatives (N05C), antidepressants (N06A), and antidementia drugs (N06D). Severity of dementia was assessed using the Reisberg GDS.[15]

\section{Intervention, Training, and Implementation}

The intervention condition involved implementation of the STA OP! protocol; all healthcare professionals (nursing staff, physicians, psychologists, physiotherapists) working on units of the intervention condition received a comprehensive stepwise multidisciplinary training of five meetings lasting 3 hours each to implement the STA OP! protocol. In these five meetings, the team members were trained in the stepwise working method of the protocol and in enhanced physical and affective assessment skills that target unmet needs commonly found in individuals with advanced dementia. A detailed description of the steps is published elsewhere.[13] To promote use of the protocol in practice, the protocol was linked to structured daily or weekly team meetings, and focus groups were formed within the units of the institution to facilitate implementation. In addition to these efforts, the project coordinator (MP) performed site visits once a week, conducted fidelity checks with nursing staff and elderly care physicians regarding their use of the STA OP! protocol, and answered their questions regarding pain or affective discomfort. Residents with moderate to severe dementia and challenging behavior were assessed and treated using the protocol. Depending on the intervention chosen, a decision was made as to how and when to proceed to the subsequent step, but in general, when effects were lacking or were limited, the intervention did not take longer than 1 week (Table 1).

\section{[TABLE 1]}

Healthcare professionals working on units in the control condition also received training, but this training lacked the stepwise component and focused on general nursing skills, dementia management, and pain. The project coordinator (MP) visited all units in the control condition once a week to provide general information on 
Pieper, M.J.C., Francke, A.L., Steen, J.T. van der, Scherder, E.J.A., Twisk, J.W.R., Kovach, C.R Achterberg, W.P. Effects of a stepwise multidisciplinary intervention for challenging behavior in ) advanced dementia: a cluster randomized controlled trial. Journal of the American Geriatries 7 Society: 2016, 64(2), 261-269

challenging behavior and dementia management and to answer staff's questions pertaining to participation in the study.

All elderly care physicians responsible for the control and intervention units received additional training from an expert physician (WA) based on the current guidelines for pain and behavior issued by the Dutch Association of Elderly Care Physicians and Social Geriatricians.[26]

\section{Statistical Analysis}

It was calculated that a sample of 168 residents was needed to detect a difference of $15 \%$ with $\alpha=0.05$ and $1-\beta=0.80$ and to account for an estimated design effect of 1.5 (cluster randomization)[27] and an expected dropout rate of $50 \%$ due to death or transfer to another nursing home unit or hospital. Differences between the intervention and control group in resident baseline characteristics were tested using the chi-square test for categorical variables, Mann-Whitney U-test for continuous variables with skewed distributions, and one-way analysis of variance for normally distributed continuous variables. Given the hierarchical data structure, multilevel analyses were used for continuous outcomes and logistic generalized estimating equation (GEE) analyses for dichotomous outcomes. Logistic GEE analysis was preferred to logistic multilevel analysis because of the instability of the latter and because the observations in the higher levels of clustering were not expected to be highly correlated.[28] Although some scores seemed to be skewed, parametric analyses were allowed, because the analyses adjusted for baseline values led to normally distributed residuals. Analyses were conducted on an intention-to-treat basis. Multilevel analyses were conducted according to a five-level structure (institute, nursing home, specialized care unit, resident, and time) and performed using MLwiN version 2.26 (University of Bristol, Bristol, UK).[29] All other analyses were performed using IBM SPSS version 21.0 (IBM Corp., Armonk, NY). The overall treatment effect over time was first investigated, and then the effect at the different time points was evaluated. For the latter, time and the interaction between treatment and time were added to the model. For both analyses, in addition to an unadjusted analysis including a correction for the baseline value of the primary outcome, an adjusted analysis was also performed. In the adjusted analysis, adjustments were made for dementia severity (moderate to severe, GDS scores 5, 6, 7), demographic characteristics (age, sex), medication use at baseline (antidepressants, antipsychotics), and comorbidity and functioning at baseline. The estimated main effects of treatment at different assessment points under these different models are reported as average changes or differences or as odds ratios (ORs).

\section{RESULTS}

\section{Participants}

The CONSORT flowchart for this study is presented in Figure 1. From the eligible pool of nursing homes, 12 nursing homes with 21 units were eligible for participation, and an independent researcher randomly assigned them. From these 21 units, 363 residents were eligible for participation. Of these 363 eligible residents, 56 $(15.4 \%)$ were not willing to participate, $13(3.6 \%)$ died, three $(0.8 \%)$ did not meet the inclusion criteria, and three $(0.8 \%)$ transferred to another nursing home or institution. 
Pieper, M.J.C., Francke, A.L., Steen, J.T. van der, Scherder, E.J.A., Twisk, J.W.R., Kovach, C.R Achterberg, W.P. Effects of a stepwise multidisciplinary intervention for challenging behavior in ) advanced dementia: a cluster randomized controlled trial. Journal of the American Geriatries 7 Society: 2016, 64(2), 261-269

\section{[FIGURE 1]}

Finally, 288 residents were included in the STA OP! trial, with 148 in the intervention condition and 140 in the control condition. Of the 148 residents in the intervention condition, $39 \%$ were analyzed using the STA OP! protocol. The mean number of steps assessed was $2.8 \pm 1.2$.

Demographic and clinical characteristics of the sample are presented in Table 2. The residents in the intervention condition were less severely impaired and took fewer antidepressants than residents in the control condition but had more symptoms of depression. During the 6-month study period, 29 residents in the control group and 30 in the intervention group were lost to follow-up (died or transferred to another unit or institution) $(P=.93)$ (Figure 1).

\section{[TABLE 2]}

\section{Effect of the Intervention}

Multilevel analyses of the NPI-NH, CMAI, CSDD, and MDS-DRS were conducted according to the intention-to-treat principle. As expected, correlations at the higher levels were close to 0 , and therefore only clustering on the specialized care unit (intraclass correlation coefficient $(\mathrm{ICC})=0.10)$ and resident $(\mathrm{ICC}=0.70)$ was taken into account in the multilevel analyses. Table 3 presents the results of the unadjusted and adjusted linear longitudinal multilevel regression analyses. Logistic GEEs were used to analyze the effect of the intervention on psychotropic medication use. Table 4 shows the results of the unadjusted and adjusted logistic GEE analyses. Results of the per-protocol analysis indicate a trend in the same direction as the intention-totreat analysis; residents treated with the STA OP! protocol had less-challenging behavior and fewer symptoms of depression than those in the control condition. Persons with dementia residing on the ward may benefit even more from the analysis and management of unmet needs of their fellow residents (snowball effect) (data not shown).

\section{[TABLE 3][TABLE 4]}

\section{Effects on Agitation and Neuropsychiatric Symptoms}

The overall adjusted mean difference in the primary outcome measure, the CMAI, was -4.07 points $(95 \%$ confidence interval $(\mathrm{CI})=-7.90$ to $-0.24, \mathrm{P}=.02)$, favoring the intervention condition; this could be explained by an effect at 3 months (post intervention/ implementation). The adjusted mean difference was -4.59 points $(95 \%$ $\mathrm{CI}=-8.70$ to $-0.48, \mathrm{P}=.01)$ at 3 months and $-3.45(95 \% \mathrm{CI}=-7.68-0.78, \mathrm{P}=.05)$ at 6 months. The overall adjusted mean difference in the NPI-NH was -3.57 points $(95 \% \mathrm{CI}=-6.30$ to $-0.84, \mathrm{P}=.005)$, also favoring the intervention condition; this could be explained by an effect at 6 months. Adjusted mean difference was -1.80 points $(95 \% \mathrm{CI}=-4.85$ to $1.25, \mathrm{P}=.12)$ at 3 months and $-5.70(95 \% \mathrm{CI}=-8.88$ to $-2.52, \mathrm{P}<.001)$ at 6 months.

\section{Effects on Depression}

The overall adjusted mean difference on the CSDD was -1.59 points $(95 \% \mathrm{CI}=$ -2.49 to $-0.69, P<.001)$, and the overall adjusted mean difference on the MDS- 
Pieper, M.J.C., Francke, A.L., Steen, J.T. van der, Scherder, E.J.A., Twisk, J.W.R., Kovach, C.R Achterberg, W.P. Effects of a stepwise multidisciplinary intervention for challenging behavior in ) advanced dementia: a cluster randomized controlled trial. Journal of the American Geriatries 7 Society: 2016, 64(2), 261-269

DRS was -0.96 points $(95 \% \mathrm{CI}=-1.40$ to $-0.52, P<.001)$, both favoring the intervention condition and significant at all time-points.

\section{Effects on Medication}

Intervention participants were significantly less likely than control participants to receive antidepressants $(\mathrm{N} 06 \mathrm{~A})(\mathrm{OR}=0.32,95 \% \mathrm{CI}=0.10-0.98)$. They were also slightly less likely to receive antipsychotics (N05A) $(\mathrm{OR}=0.69,95 \% \mathrm{CI}=0.30$ $1.60)$, anxiolytics $(\mathrm{N} 05 \mathrm{~B})(\mathrm{OR}=0.75,95 \% \mathrm{CI}=0.27-2.12)$, and hypnotics and sedatives $(\mathrm{N} 05 \mathrm{C})(\mathrm{OR}=0.90,95 \% \mathrm{CI}=0.34-2.37)$ and slightly more likely to receive antidementia drugs $(\mathrm{N} 06 \mathrm{D})(\mathrm{OR}=2.63,95 \% \mathrm{CI}=0.63-10.95)$, although these differences were not significant. Residents in the intervention condition were less likely to receive psychotropic medication but slightly, but not significantly so, more likely to receive anxiolytics at 3 months $(\mathrm{OR}=1.12,95 \% \mathrm{CI}=0.37-3.35)$ and hypnotics and sedatives (N05C) at 6 months $(\mathrm{OR}=1.13,95 \% \mathrm{CI}=0.36-3.50)$.

\section{DISCUSSION}

In this cluster randomized controlled trial, implementation of the adjusted and redesigned version of the serial trial intervention STA OP! improved overall agitation, depression, and other neuropsychiatric symptoms, showing that a customized stepwise intervention focusing on psychosocial and physical unmet needs is effective for nursing home residents with dementia and that these effects can be achieved without an increase in psychotropic drug use. Only at 3 months was a slight increase found in anxiolytic drug use; this is probably explained by an earlier identification of challenging behavior (intervention effect). In view of the rapidly increasing number of people with advanced dementia, this provides a hopeful message. Also, because there is no cure for dementia, every attempt should be made to improve the quality of life of these individuals. Current epidemiological reports forecast a considerable growth in the number of people with dementia; [30] the burdens and challenges of caring for this group are formidable. In dementia, agitation, depression, and other neuropsychiatric symptoms present a major challenge for informal caregivers, neurologists, psychiatrists, geriatricians, and longterm care staff. This customized, stepwise protocol, based on the STI, appears to be beneficial in these complex clinical situations.

Although the hypothesized decrease in psychotropic medication was confirmed, statistical significance was not reached. One explanation for this might be that this trial had insufficient power to detect significant differences, because the power calculations were based on the primary outcome - the CMAI. Another explanation could be that the physicians were not coached enough in decreasing psychotropic medication when starting the STA OP! protocol, despite the additional training they received regarding the prescription of psychotropic medications and their use in daily practice. Nevertheless, the total effect of the program can be considered clinically relevant, because the effects on behavior were within the range that other researchers investigating antipsychotic drugs have reported.[31-33]

This trial has several limitations. First, although the primary source of information was the behavior of the resident, who was unaware of being included in the intervention or control condition, and the trained research assistant who collected the information was blinded for the condition, the outcome measurement was based on the observations of the nurses and could not be blinded. The possibility of bias, or Hawthorn effect, can therefore not be excluded. Second, after implementation, the 
Pieper, M.J.C., Francke, A.L., Steen, J.T. van der, Scherder, E.J.A., Twisk, J.W.R., Kovach, C.R Achterberg, W.P. Effects of a stepwise multidisciplinary intervention for challenging behavior in ) advanced dementia: a cluster randomized controlled trial. Journal of the American Geriatries 7 Society: 2016, 64(2), 261-269

intervention was performed in only a small proportion of the residents, despite the fact that all residents met the inclusion criteria and were eligible for treatment with STA OP! (39\% (58/148) of the residents in the intervention group started STA OP!). This raises questions about the fidelity procedures and the knowledge, time, and resource requirements to implement a multicomponent intervention (such as the STI or STA OP!) in a classical randomized trial. In a short period of time, the staff was expected to assess behavior, environmental stimuli, and the unmet physical and psychosocial needs of almost all residents on the ward and to implement an appropriately customized intervention trial in addition to their daily workload. Despite the comprehensive education and fidelity program, to control for threats to construct and internal validity, the disappointing level of implementation suggests that future research should examine the feasibility of implementation in a nursing home setting. It can be argued that, in the present study, the inclusion threshold was set too low because almost all participants qualified as having challenging behavior or pain. This study suggests that future research should identify residents with neuropsychiatric symptoms that are particularly severe or burdensome for the resident and are likely to be modified using a stepwise protocol that emphasizes nonpharmacological interventions.

In addition to the above-mentioned limitations, this trial has several strengths: the use of several reliable and valid instruments for a broad range of neuropsychiatric symptoms, including depression, and the cluster-randomized design and sound methodological multilevel analysis approach, which accounted for clustering of the data. The latter is particularly important when studying behavior on care units for dementia because behavioral problems are largely related to environmental, rather than individual, characteristics.[34]

\section{CONCLUSION}

This cluster randomized trial showed that the STA OP! (a stepwise multidisciplinary intervention) is effective in reducing neuropsychiatric symptoms and the use of psychotropic medication in nursing home residents with advanced dementia.

\section{Acknowledgments}

The authors thank all the healthcare professionals and the management and staff of the participating nursing homes for their collaboration.

Conflict of Interest: All authors declare no support from any organization for the submitted work, no financial relationships with any organization that might have an interest in the submitted work in the previous 3 years, and no other relationships or activities that could appear to have influenced the submitted work.

Author Contributions: Marjoleine J.C. Pieper drafted the manuscript, coordinated the data collection, and analyzed the results. Anneke L. Francke, Wilco P. Achterberg, Erik J.A. Scherder, Christine R. Kovach, and Jenny T. van der Steen designed the STA OP! trial and helped to draft the manuscript. All authors were involved in revising the manuscript and read and approved the final version of the manuscript. 
Pieper, M.J.C., Francke, A.L., Steen, J.T. van der, Scherder, E.J.A., Twisk, J.W.R., Kovach, C.R Achterberg, W.P. Effects of a stepwise multidisciplinary intervention for challenging behavior in advanced dementia: a cluster randomized controlled trial. Journal of the American Geriatries 7 Society: 2016, 64(2), 261-269

Sponsor's Role: The funding source (Innovatiefonds Zorgverzekeraars) had no role in the study design, data collection, data analysis, or writing of the report. The corresponding author and all coauthors had full access to the study data and had final responsibility for the decision to submit for publication.

\section{REFERENCES}

1. Ballard CG, O'Brien JT, Swann AG et al. The natural history of psychosis and depression in dementia with Lewy bodies and Alzheimer's disease: Persistence and new cases over 1 year of follow-up. J Clin Psychiatry 2001;62: 46-49.

2. Brodaty $\mathrm{H}$, Connors $\mathrm{MH}, \mathrm{Xu} \mathrm{J}$ et al. Predictors of institutionalization in dementia: A three year longitudinal study. J Alzheimers Dis 2014;40:221- 226.

3. Luppa M, Luck T, Weyerer S et al. Prediction of institutionalization in the elderly. A systematic review. Age Ageing 2010;39:31-38.

4. Gaugler JE, Yu F, Krichbaum K et al. Predictors of nursing home admission for persons with dementia. Med Care 2009;47:191-198.

5. Wetzels RB, Zuidema SU, de Jonghe JF et al. Course of neuropsychiatric symptoms in residents with dementia in nursing homes over 2-year period. Am J Geriatr Psychiatry 2010;18:1054-1065.

6. Briesacher BA, Limcangco MR, Simoni-Wastila $L$ et al. The quality of antipsychotic drug prescribing in nursing homes. Arch Intern Med 2005;165:1280-1285.

7. Steinberg M, Corcoran C, Tschanz JT et al. Risk factors for neuropsychiatric symptoms in dementia: The Cache County Study. Int J Geriatr Psychiatry 2006;21:824-830.

8. Cohen-Mansfield J. Nonpharmacologic treatment of behavioral disorders in dementia. Curr Treat Options Neurol 2013;15:765-785.

9. Pieper MJ, van Dalen-Kok AH, Francke AL et al. Interventions targeting pain or behaviour in dementia: A systematic review. Ageing Res Rev 2013;12:1042-1055.

10. Kovach CR, Noonan PE, Schlidt AM et al. The Serial Trial Intervention: An innovative approach to meeting needs of individuals with dementia. J Gerontol Nurs 2006;32:18-25; quiz 26-27.

11. Conroy S, Van Der Cammen T, Schols J et al. Medical services for older people in nursing homes-comparing services in England and the Netherlands. J Nutr Health Aging 2009;13:559-563.

12. Ribbe MW, Ljunggren $G$, Steel $K$ et al. Nursing homes in 10 nations: $A$ comparison between countries and settings. Age Ageing 1997;26 (Suppl 2):3-12.

13. Pieper MJC, Achterberg WP, Francke AL et al. The implementation of the serial trial intervention for pain and challenging behaviour in advanced dementia patients (STA OP!): A clustered randomized controlled trial. BMC Geriatr 2011;11:12.

14. Koopmans RT, Lavrijsen JC, Hoek F. Concrete steps toward academic medicine in long term care. J Am Med Dir Assoc 2013;14:781-783.

15. Reisberg B, Ferris SH, de Leon MJ et al. The Global Deterioration Scale for assessment of primary degenerative dementia. Am J Psychiatry 1982;139:1136-1139.

16. Cohen-Mansfield J, Marx MS, Rosenthal AS. A description of agitation in a nursing home. J Gerontol 1989;44:M77-M84.

17. Cummings JL, Mega M, Gray K et al. The Neuropsychiatric Inventory: Comprehensive assessment of psychopathology in dementia. Neurology 1994;44:2308-2314.

18. de Jonghe JF, Kat MG. Factor structure and validity of the Dutch version of the CohenMansfield Agitation Inventory (CMAI-D). J Am Geriatr Soc 1996;44:888-889.

19. Zuidema SU, de Jonghe JF, Verhey FR et al. Agitation in Dutch institutionalized patients with dementia: Factor analysis of the Dutch version of the Cohen-Mansfield Agitation Inventory. Dement Geriatr Cogn Disord 2007;23:35-41.

20. Cohen-Mansfield J, Libin A. Assessment of agitation in elderly patients with dementia: Correlations between informant rating and direct observation. Int J Geriatr Psychiatry 2004;19:881-891. 
Pieper, M.J.C., Francke, A.L., Steen, J.T. van der, Scherder, E.J.A., Twisk, J.W.R., Kovach, C.R Achterberg, W.P. Effects of a stepwise multidisciplinary intervention for challenging behavior in ) advanced dementia: a cluster randomized controlled trial. Journal of the American Geriatries 7 Society: 2016, 64(2), 261-269

21. Wood S, Cummings JL, Hsu MA et al. The use of the neuropsychiatric inventory in nursing home residents. Characterization and measurement. Am J Geriatr Psychiatry 2000;8:75-83.

22. Kat MG, de Jonghe JF, Aalten $P$ et al. [Neuropsychiatric symptoms of dementia: psychometric aspects of the Dutch Neuropsychiatric Inventory (NPI)]. Tijdschr Gerontol Geriatr 2002;33:150-155.

23. Alexopoulos GS, Abrams RC, Young RC et al. Cornell Scale for Depression in Dementia. Biol Psychiatry 1988;23:271-284.

24. Burrows AB, Morris JN, Simon SE et al. Development of a Minimum Data Set-based depression rating scale for use in nursing homes. Age Ageing 2000;29:165-172.

25. WHO Collaborating Center for Drug Statistics Methodology [on-line].

Available at http://www.whocc.no/atc_ddd_methodology/history/ Accessed February 1, 2014.

26. van Kleef M, Geurts JW. Useful guideline for treatment of pain in vulnerable elderly people. Ned Tijdschr Geneeskd 2012;155:A4933.

27. Eldridge SM, Ashby D, Kerry S. Sample size for cluster randomized trials: Effect of coefficient of variation of cluster size and analysis method. Int J Epidemiol 2006;35:12921300.

28. Twisk JWR. Applied Longitudinal Data Analysis for Epidemiology, 2nd Ed. Cambridge, UK: Cambridge University Press, 2013.

29. Rasbash J, Charlton C, Browne WJ et al. MLwiN Version 2.1. Bristol, UK: Centre for Multilevel Modelling, University of Bristol, 2009.

30. Prince M, Bryce R, Albanese $E$ et al. The global prevalence of dementia: A systematic review and metaanalysis. Alzheimers Dement 2013;9:63.e62- 75.e62.

31. Ballard CG, Gauthier S, Cummings JL et al. Management of agitation and aggression associated with Alzheimer disease. Nat Rev Neurol 2009;5:245- 255.

32. Porsteinsson AP, Drye LT, Pollock BG et al. Effect of citalopram on agitation in Alzheimer disease: The CitAD randomized clinical trial. JAMA 2014;311:682-691.

33. Fossey J, Ballard C, Juszczak E et al. Effect of enhanced psychosocial care on antipsychotic use in nursing home residents with severe dementia: Cluster randomised trial. BMJ 2006;332:756-761.

34. Zuidema SU, de Jonghe JF, Verhey FR et al. Psychotropic drug prescription in nursing home patients with dementia: Influence of environmental correlates and staff distress on physicians' prescription behavior. Int Psychogeriatr 2011;23:1632-1639. 
Pieper, M.J.C., Francke, A.L., Steen, J.T. van der, Scherder, E.J.A., Twisk, J.W.R., Kovach, C.R Achterberg, W.P. Effects of a stepwise multidisciplinary intervention for challenging behavior in ) advanced dementia: a cluster randomized controlled trial. Journal of the American Geriatries 7 Society: 2016, 64(2), 261-269

\section{TABLES AND FIGURES}

Table 1. Description of STA OP! Steps

\begin{tabular}{|l|l|}
\hline Step & \multicolumn{1}{c|}{ Description } \\
\hline \hline 1. & Nurses, nursing home physicians, and healthcare professionals (multidisciplinary team) should \\
identify behavioral symptoms using an explicit schedule and procedures. When a resident \\
exhibits changes in behavior that are not effectively treated, and basic care provided is checked \\
at step 0, the nurse initiates the STA OP! at step 1. The STA OP! process is stopped when \\
behavioral symptoms decrease by 50\% or more. Continued movement through steps of the \\
STA OP! is based on results of assessments and decreases in symptoms in time frames that \\
have been established for specified treatments. If behavioral symptoms continue after \\
completing these five steps, the process is repeated at the initial behavioral change \\
identification.
\end{tabular}

Start with a behavioral change identification; define the target behavior, its expression and when (in what situation) this behavior is challenging. Check if the behavior is new or recurrent. If the behavior is recurrent, check what has been done in the past to treat it. Define for whom the behavior is challenging: the patient, family, or caregivers? A psychologist can be consulted at this step.

If the nurses and the multidisciplinary team of healthcare professionals make a clear description of the targeted behavior, the nurse moves to the next step (0).

Perform a basic care needs assessment and determine whether basic care needs are fulfilled (e.g., hunger, thirst, eyeglasses, hearing aids, toileting).

0

If assessment is positive, a targeted intervention is implemented, or the appropriate discipline is consulted to begin treatment. If the assessment is negative, or if treatment fails to decrease symptoms, the nurse moves to the next step (1).

Perform a pain and physical needs assessment. In addition to a brief physical nursing assessment by the nursing home physician, nurses fill out an observational pain instrument (Dutch Pain Assessment Checklist for Seniors with Limited Ability to Communicate). This form is given to the nursing home physician (or if available a nurse practitioner), who performs a morecomprehensive physical assessment to find other probable physical causes associated with discomfort. For residents already using pain medication or psychotropic drugs who still have behavioral symptoms possibly related to pain or affective discomfort, the nursing home physician assesses whether the medication given is in accordance with the guidelines of the World Health Organization and Verenso (the Dutch association of nursing home physicians) (also see steps 4 and 5).

If assessment is positive, a targeted intervention is implemented, or the appropriate discipline is consulted to begin treatment. If the assessment is negative, or if treatment fails to decrease symptoms, the nurse moves to the next step (2).

Perform affective needs assessment that focuses on needs of people with dementia: (a)

2 environmental stress threshold not exceeded, (b) balance between sensory-stimulating and sensory-calming activity throughout the day, and (c) receipt of meaningful human interaction 
Pieper, M.J.C., Francke, A.L., Steen, J.T. van der, Scherder, E.J.A., Twisk, J.W.R., Kovach, C.R Achterberg, W.P. Effects of a stepwise multidisciplinary intervention for challenging behavior in ) advanced dementia: a cluster randomized controlled trial. Journal of the American Geriatries 7 Society: 2016, 64(2), 261-269

\begin{tabular}{|c|c|}
\hline Step & Description \\
\hline & $\begin{array}{l}\text { each day. The psychologist (or social worker) working in the nursing home can be consulted at } \\
\text { this step. }\end{array}$ \\
\hline & $\begin{array}{l}\text { If assessment is positive, a targeted intervention is implemented, or the appropriate discipline is } \\
\text { consulted to begin treatment. If the assessment is negative, or if treatment fails to decrease } \\
\text { symptoms, the nurse moves to the next step (3). }\end{array}$ \\
\hline & $\begin{array}{l}\text { Administer a trial of nonpharmacological comfort treatment(s). Treatments used are customized } \\
\text { to the person and the situation and are based on a list of psychosocial and environmental } \\
\text { treatments that have been associated with decreasing agitated behaviors. }\end{array}$ \\
\hline 3 & $\begin{array}{l}\text { If a one-time treatment is effective, and continued use is desirable, take actions needed to ensure } \\
\text { continued treatment (e.g., communicate new treatment to other staff and family, write it down in } \\
\text { the patient's care plan with prescribed times or administration). If a trial of nonpharmacological } \\
\text { comfort treatment(s) does not ameliorate behaviors in a time frame likely to show outcomes, the } \\
\text { nurse should move to the next step (4). }\end{array}$ \\
\hline & $\begin{array}{l}\text { Administer a trial of analgesic agents by administering the prescribed as-needed analgesic agent } \\
\text { or obtaining orders to escalate a current analgesic medication. }\end{array}$ \\
\hline 4 & $\begin{array}{l}\text { If treatment is effective, and continued use is desirable, take actions needed to ensure continued } \\
\text { treatment (e.g., schedule dosing of effective treatments for continued use, write it down in the } \\
\text { patient's care plan with prescribed times or administration). If there is not a response to a trial } \\
\text { course of analgesic medications, consider consultation regarding further escalation or proceed to } \\
\text { the next step (5). Stop ineffective treatments. }\end{array}$ \\
\hline & $\begin{array}{l}\text { Consult with other disciplines (e.g., psychiatrist) or administer a trial of prescribed as-needed } \\
\text { psychotropic drugs in this step if the behavior continues, alternatives are carefully considered, } \\
\text { and potential side effects are weighed against the comfort needs of the resident. }\end{array}$ \\
\hline 5 & $\begin{array}{l}\text { Monitor for recurrence and new problems. Conduct regular comprehensive assessments. } \\
\text { Establish clear criteria for evaluation of problems and treatment effectiveness, need for }\end{array}$ \\
\hline
\end{tabular}


Pieper, M.J.C., Francke, A.L., Steen, J.T. van der, Scherder, E.J.A., Twisk, J.W.R., Kovach, C.R Achterberg, W.P. Effects of a stepwise multidisciplinary intervention for challenging behavior in advanced dementia: a cluster randomized controlled trial. Journal of the American Geriateies 7 Society: 2016, 64(2), 261-269

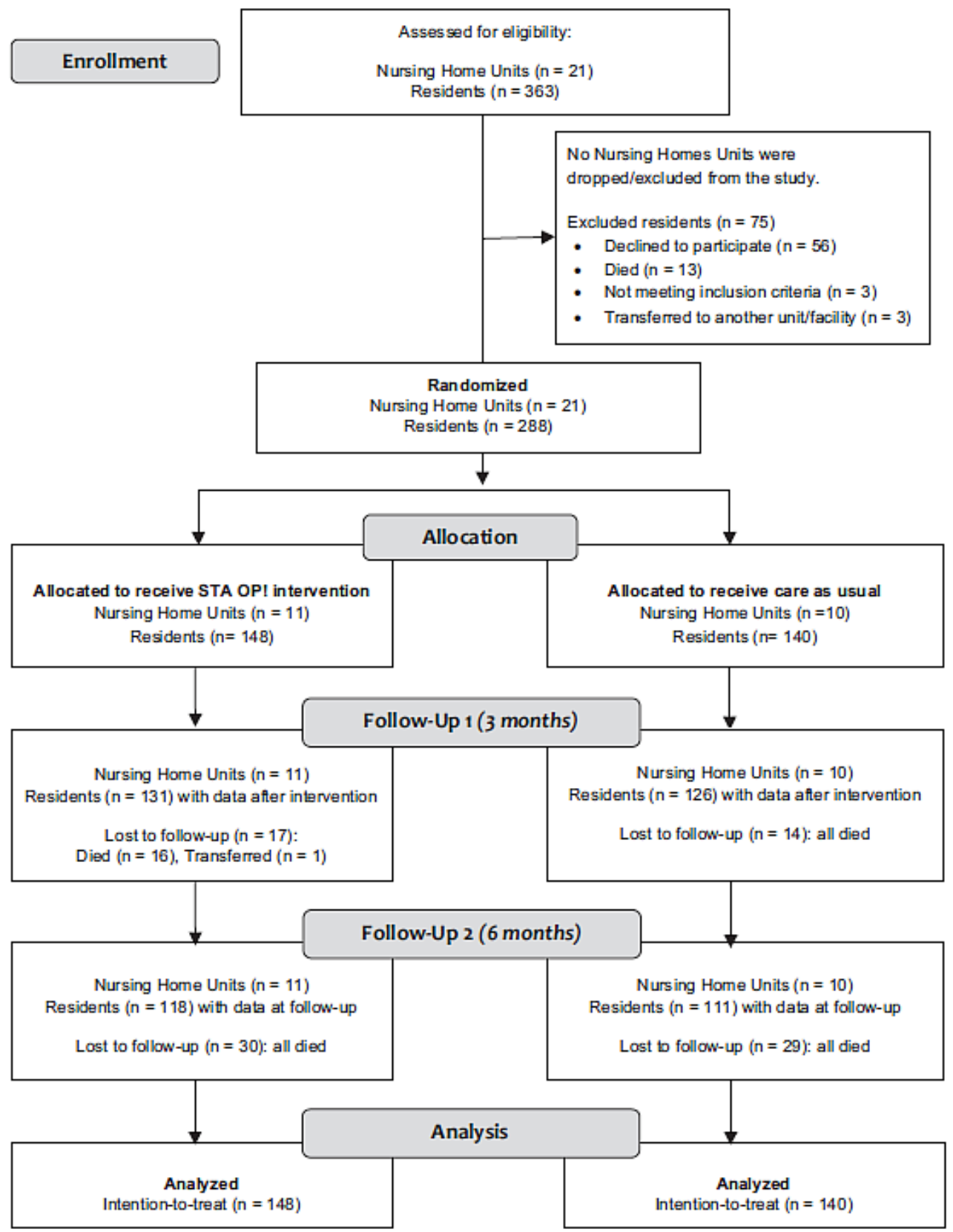

Figure 1. STA OP! flowchart. 
Pieper, M.J.C., Francke, A.L., Steen, J.T. van der, Scherder, E.J.A., Twisk, J.W.R., Kovach, C.R Achterberg, W.P. Effects of a stepwise multidisciplinary intervention for challenging behavior in ) advanced dementia: a cluster randomized controlled trial. Journal of the American Geriatries 7

Society: 2016, 64(2), 261-269

Table 2. Baseline Characteristics of the STA OP! Sample

\begin{tabular}{|c|c|c|c|}
\hline Characteristic & Control, $n=140$ & \begin{tabular}{|c|} 
Intervention, \\
$\mathrm{n}=148$ \\
\end{tabular} & $P$-Value \\
\hline Age, mean \pm SD & $83.3 \pm 6.9$ & $84.3 \pm 7.4$ & $.25 \mathrm{a}$ \\
\hline \multicolumn{4}{|l|}{ Sex, n (\%) } \\
\hline Male & $40(28.6)$ & $41(27.7)$ & $.87 \mathrm{~b}$ \\
\hline Female & $100(71.4)$ & $107(72.3)$ & \\
\hline \multicolumn{4}{|c|}{ Severity of cognitive decline (Reisberg Global Deterioration Scale stage (range 1-7)), n (\%) } \\
\hline Moderately severe (5) & $12(8.6)$ & $23(15.5)$ & $.04 \mathrm{~b}$ \\
\hline Severe (6) & $81(57.9)$ & $92(62.2)$ & \\
\hline Very severe $(7)$ & $47(33.6)$ & $33(22.3)$ & \\
\hline \multicolumn{4}{|c|}{ Type of medication (Anatomical Therapeutic Chemical code), $\mathrm{n}(\%) \mathrm{d}$} \\
\hline \multicolumn{4}{|l|}{ Psycholeptic medications (N05) } \\
\hline No psycholeptic medication (N05) & $53(38.4)$ & $57(39.6)$ & $.84 \mathrm{~b}$ \\
\hline Antipsychotics (N05A) & $51(37.0)$ & $51(35.4)$ & $.79 b$ \\
\hline Anxiolytics (N05B) & $43(31.2)$ & $53(36.8)$ & $.32 \mathrm{~b}$ \\
\hline Hypnotics or sedatives (N05C) & $32(23.2)$ & $24(16.7)$ & $.17 \mathrm{~b}$ \\
\hline \multicolumn{4}{|l|}{ Psychoanaleptic medications (N06) } \\
\hline No psychoanaleptic medication (N06) & $93(67.4)$ & $108(75.0)$ & $.16 \mathrm{~b}$ \\
\hline Antidepressants (N06A) & $40(28.9)$ & $27(18.8)$ & $.04 \mathrm{~b}$ \\
\hline Antidementia drugs (N06D) & $14(10.1)$ & $11(7.6)$ & $.46 \mathrm{~b}$ \\
\hline \multicolumn{4}{|l|}{ Minimum Dataset Comorbidity subgroup, n (\%) } \\
\hline $\begin{array}{l}\text { Circulatory system (e.g., arteriosclerotic heart } \\
\text { disease, arteriosclerosis, thrombosis, high or low } \\
\text { blood pressure) }\end{array}$ & $76(54.3)$ & $76(51.4)$ & $45 b$ \\
\hline $\begin{array}{l}\text { Respiratory system (e.g., chronic obstructive } \\
\text { pulmonary disease, emphysema, asthma) }\end{array}$ & $12(8.6)$ & $18(12.2)$ & $32 b$ \\
\hline $\begin{array}{l}\text { Locomotor system (e.g., (rheumatoid) arthritis, } \\
\text { osteoporosis, column or malign fracture, } \\
\text { amputation) }\end{array}$ & $41(29.3)$ & $34(23.0)$ & $45 b$ \\
\hline $\begin{array}{l}\text { Nervous system (e.g., cerebrovascular accident, } \\
\text { stroke, hemorrhage, aphasia, epilepsy, hemiplegia, } \\
\text { cerebral palsy) }\end{array}$ & $39(27.9)$ & $32(21.6)$ & $.39 b$ \\
\hline $\begin{array}{l}\text { Endocrine, metabolic system (e.g., diabetes } \\
\text { mellitus, hyper- or hypothyroid) }\end{array}$ & $42(30.0)$ & $36(24.2)$ & $.28 b$ \\
\hline $\begin{array}{l}\text { Sensory system (e.g., cataract, diabetes mellitus, } \\
\text { retinopathy, glaucoma, macular degeneration) }\end{array}$ & $31(22.1)$ & $21(14.2)$ & $.25 b$ \\
\hline $\begin{array}{l}\text { Infection (e.g., urinary tract }<30 \text { days, pneumonia, } \\
\text { airway infection) }\end{array}$ & $9(6.4)$ & $9(6.1)$ & $.90 b$ \\
\hline
\end{tabular}


Pieper, M.J.C., Francke, A.L., Steen, J.T. van der, Scherder, E.J.A., Twisk, J.W.R., Kovach, C.R Achterberg, W.P. Effects of a stepwise multidisciplinary intervention for challenging behavior in ) advanced dementia: a cluster randomized controlled trial. Journal of the American Geriatries 7 Society: 2016, 64(2), 261-269

\begin{tabular}{|c|c|c|c|}
\hline Characteristic & Control, $n=140$ & $\begin{array}{c}\text { Intervention, } \\
\mathbf{n}=\mathbf{1 4 8} \\
\end{array}$ & $P$-Value \\
\hline $\begin{array}{l}\text { Other (allergies, cancer, anemia, kidney } \\
\text { insufficiency) }\end{array}$ & $20(14.3)$ & $22(14.9)$ & $.99 b$ \\
\hline \multicolumn{4}{|l|}{ Behavior, mean \pm SDe } \\
\hline $\begin{array}{l}\text { Cohen-Mansfield Agitation Inventory (range 29- } \\
203 \text { ) }\end{array}$ & $47.7 \pm 19.0$ & $46.0 \pm 17.2$ & $.48 \mathrm{c}$ \\
\hline $\begin{array}{l}\text { Neuropsychiatric Inventory-Nursing Home } \\
\text { version (range 0-144) }\end{array}$ & $14.3 \pm 12.9$ & $17.0 \pm 16.4$ & $.24 c$ \\
\hline \multicolumn{4}{|l|}{ Mood, mean \pm SDe } \\
\hline $\begin{array}{l}\text { Cornell Scale for Depression in Dementia (range 0- } \\
\text { 38) }\end{array}$ & $5.0 \pm 3.7$ & $6.7 \pm 4.0$ & $<.001 \mathrm{c}$ \\
\hline $\begin{array}{l}\text { Minimum Dataset-Depression Rating Scale (range } \\
0-14 \text { ) }\end{array}$ & $1.8 \pm 2.0$ & $2.2 \pm 2.2$ & $.06 \mathrm{c}$ \\
\hline $\begin{array}{l}\text { Functioning (Katz activity of daily living scale } \\
\text { (range 6-24)), mean } \pm \text { SDe }\end{array}$ & $18.0 \pm 5.2$ & $16.5 \pm 5.2$ & $.01 \mathrm{c}$ \\
\hline
\end{tabular}

${ }^{\mathrm{a}}$ One-way analysis of variance.

${ }^{\mathrm{b}}$ Pearson chi-square.

${ }^{c}$ Mann-Whitney U-test.

${ }^{\mathrm{d}} \mathrm{N}=282$ (control, $\mathrm{n}=138$; intervention, $\mathrm{n}=144$ ).

${ }^{\mathrm{e}}$ Higher scores indicate more symptoms of depression, more-challenging behavior, or greater functional decline.

$\mathrm{SD}=$ standard deviation.

Table 3. Average Change in Challenging Behavior (Cohen-Mansfield Agitation Inventory (CMAI), Neuropsychiatric Inventory Nursing Home version (NPI-NH)) and Symptoms of Depression (Cornell Depression Scale in Dementia (CSDD), Minimum Dataset Depression Rating Scale (MDS-DRS)) 3 and 6 Months After the Intervention

\begin{tabular}{|c|c|c|}
\hline \multirow{2}{*}{ Outcome Measure } & Unadjusted (Crude) Model & Adjusted Model \\
\hline & \multicolumn{2}{|c|}{ ק (95\% Confidence Interval) $P$-Value } \\
\hline \multicolumn{3}{|l|}{ CMAI } \\
\hline Overall & $-3.48(-7.05-0.10) .06$ & $-4.07(-7.90$ to -0.24$) .02$ \\
\hline 3 months & $-4.12(-8.00$ to -0.25$) .04$ & $-4.59(-8.70$ to -0.48$) .01$ \\
\hline 6 months & $-2.72(-6.72-1.28) .18$ & $-3.45(-7.68-0.78) .05$ \\
\hline \multicolumn{3}{|l|}{ NPI-NH } \\
\hline Overall & $-3.14(-5.64$ to -0.65$) .01$ & $-3.57(-6.30$ to -0.84$) .005$ \\
\hline 3 months & $-1.71(-4.60-1.17) .25$ & $-1.80(-4.85-1.25) .12$ \\
\hline 6 months & $-4.85(-7.86$ to -1.83$) .002$ & $-5.70(-8.88$ to -2.52$)<.001$ \\
\hline \multicolumn{3}{|l|}{ Symptoms of depression } \\
\hline CSDD & & \\
\hline
\end{tabular}


Pieper, M.J.C., Francke, A.L., Steen, J.T. van der, Scherder, E.J.A., Twisk, J.W.R., Kovach, C.R Achterberg, W.P. Effects of a stepwise multidisciplinary intervention for challenging behavior in ) advanced dementia: a cluster randomized controlled trial. Journal of the American Geriatries 7 Society: 2016, 64(2), 261-269

\begin{tabular}{||l||c||c||}
\hline \multirow{2}{*}{ Outcome Measure } & Unadjusted (Crude) Model & \multicolumn{1}{c||}{ Adjusted Model } \\
\cline { 2 - 3 } & \multicolumn{2}{|c||}{$\boldsymbol{\beta}(\mathbf{9 5 \%}$ Confidence Interval) $\boldsymbol{P}$-Value } \\
\hline \hline Overall & $-1.49(-2.41$ to -0.56$) .002$ & $-1.59(-2.49$ to -0.69$)<.001$ \\
\hline \hline 3 months & $-1.21(-2.25$ to -0.18$) .02$ & $-1.30(-2.31$ to -0.29$) .006$ \\
\hline \hline 6 months & $-1.81(-2.89$ to -0.74$) .001$ & $-1.94(-2.99$ to -0.89$)<.001$ \\
\hline MDS-DRS & $-0.90(-1.35$ to -0.44$)<.001$ & $-0.96(-1.40$ to -0.52$)<.001$ \\
\hline \hline Overall & $-0.73(-1.23$ to -0.22$) .005$ & $-0.76(-1.25$ to -0.27$) .001$ \\
\hline \hline 3 months & $-1.09(-1.61$ to -0.57$)<.001$ & $-1.18(-1.69$ to -0.67$)<.001$ \\
\hline \hline 6 months
\end{tabular}

${ }^{1}$ Reference category for the intervention effect is the control condition. Regression coefficients $(\beta)$ reflect the average differences in challenging behavior during the 6month study period using neuropsychiatric symptoms (NPI-NH), agitation (CMAI), or symptoms of depression (CSDD, MDS-DRS) over the two follow-up periods ( 3 and 6 months after intervention) from baseline. A negative $\beta$ indicates a positive intervention effect on challenging behavior or symptoms of depression of residents in the intervention condition (STA OP! program) (3 and 6 months after the intervention).

${ }^{2} \beta$ reflects the decrease in challenging behavior or symptoms of depression during the 6-month study period in the intervention group.

${ }^{3}$ Crude model adjusted for baseline value of neuropsychiatric symptoms or agitation and levels of clustering for time, individual, and nursing home unit.Adjusted model also adjusted for sex, age, stage of dementia, comorbidity, medication use (e.g., antidepressive, antipsychotic), and baseline functioning.

Table 4. Change in Psychotropic Medication Use

\begin{tabular}{|c|c|c|}
\hline \multirow{2}{*}{$\begin{array}{c}\text { Psychotropic Medication } \\
\text { (Anatomical Therapeutic } \\
\text { Classification) }\end{array}$} & Unadjusted Model & Adjusted Model \\
\hline & \multicolumn{2}{|c|}{ Odds Ratio (95\% Confidence Interval) P-Value } \\
\hline \multicolumn{3}{|l|}{1.} \\
\hline \multicolumn{3}{|l|}{ Antipsychotics (N05A) } \\
\hline Overall & $0.68(0.32-1.46) .32$ & $0.69(0.30-1.60) .38$ \\
\hline 3 months & $0.57(0.26-1.28) .18$ & $0.55(0.23-1.34) .19$ \\
\hline 6 months & $0.83(0.35-2.01) .69$ & $0.87(0.33-2.30) .78$ \\
\hline \multicolumn{3}{|l|}{ Anxiolytics (N05B) } \\
\hline Overall & $1.10(0.50-2.40) .81$ & $0.75(0.27-2.12) .59$ \\
\hline 3 months & $1.60(0.66-3.83) .30$ & $1.12(0.37-3.35) .84$ \\
\hline 6 months & $0.61(0.24-1.56) .30$ & $0.41(0.12-1.37) .15$ \\
\hline \multicolumn{3}{|l|}{ Hypnotics and sedatives (N05C) } \\
\hline Overall & $0.88(0.36-2.15) .77$ & $0.90(0.34-2.37) .82$ \\
\hline
\end{tabular}


Pieper, M.J.C., Francke, A.L., Steen, J.T. van der, Scherder, E.J.A., Twisk, J.W.R., Kovach, C.R Achterberg, W.P. Effects of a stepwise multidisciplinary intervention for challenging behavior in ) advanced dementia: a cluster randomized controlled trial. Journal of the American Geriatries Society: 2016, 64(2), 261-269

\begin{tabular}{|c|c|c|}
\hline \multirow{2}{*}{$\begin{array}{c}\text { Psychotropic Medication } \\
\text { (Anatomical Therapeutic } \\
\text { Classification) }\end{array}$} & Unadjusted Model & Adjusted Model \\
\hline & \multicolumn{2}{|c|}{ Odds Ratio (95\% Confidence Interval) P-Value } \\
\hline 3 months & $0.79(0.32-1.92) .60$ & $0.75(0.28-2.03) .57$ \\
\hline 6 months & $1.12(0.39-3.21) .84$ & $1.13(0.36-3.50) .84$ \\
\hline \multicolumn{3}{|l|}{ Antidepressants (N06A) } \\
\hline Overall & $0.76(0.27-2.16) .60$ & $0.32(0.10-0.98) .046$ \\
\hline 3 months & $0.57(0.17-1.87) .35$ & $0.27(0.07-0.97) .04$ \\
\hline 6 months & $0.88(0.28-2.78) .82$ & $0.40(0.10-1.54) .18$ \\
\hline \multicolumn{3}{|l|}{ Antidementia drugs (N06D) } \\
\hline Overall & $1.28(0.35-4.76) .71$ & $2.63(0.63-10.95) .18$ \\
\hline 3 months & $1.41(0.40-5.00) .59$ & $3.51(0.84-14.71) .08$ \\
\hline 6 months & $0.93(0.20-4.44) .93$ & $1.87(0.30-11.46) .50$ \\
\hline
\end{tabular}

Reference category for the intervention effect is the control condition. Odds ratios (OR) reflect psychotropic medication use during the 6-month study period. OR $<1.0$ indicates a decrease in psychotropic medication use of residents with moderate to severe dementia in the intervention condition (STA OP! program) compared to residents in the control condition over time ( 3 and 6 months post intervention). Crude model adjusted for baseline value of neuropsychiatric symptoms or agitation, and levels of clustering for time, individual, and nursing home unit.

Adjusted model also adjusted for sex, age, stage of dementia, comorbidity, medication use (e.g., antidepressive, antipsychotic), and baseline functioning. 